This item was submitted to Loughborough's Research Repository by the author.

Items in Figshare are protected by copyright, with all rights reserved, unless otherwise indicated.

\title{
Performance assessment of evolutionary algorithms in power system optimization problems
}

PLEASE CITE THE PUBLISHED VERSION

http://dx.doi.org/10.1109/PTC.2015.7232622

PUBLISHER

(C) IEEE

VERSION

AM (Accepted Manuscript)

\section{PUBLISHER STATEMENT}

This work is made available according to the conditions of the Creative Commons Attribution-NonCommercialNoDerivatives 4.0 International (CC BY-NC-ND 4.0) licence. Full details of this licence are available at: https://creativecommons.org/licenses/by-nc-nd/4.0/

\section{LICENCE}

CC BY-NC-ND 4.0

\section{REPOSITORY RECORD}

Rueda, Jose L., Istvan Erlich, and Francisco M. Gonzalez-Longatt. 2019. "Performance Assessment of Evolutionary Algorithms in Power System Optimization Problems”. figshare. https://hdl.handle.net/2134/20509. 


\title{
Performance Assessment of Evolutionary Algorithms in Power System Optimization Problems
}

\author{
José L. Rueda ${ }^{1}$, István Erlich ${ }^{2}$, Francisco González-Longatt ${ }^{3}$ \\ ${ }^{1}$ Delft University of Technology, The Netherlands \\ ${ }^{2}$ University Duisburg-Essen, Germany \\ ${ }^{3}$ Loughborough University, United Kingdom \\ j.1.ruedatorres@tudelft.nl, istvan.erlich@uni-due.de,fglongatt@ieee.org
}

\begin{abstract}
Due to the stochastic nature, there are several concerns on the effectiveness and robustness of evolutionary algorithms when applied to solve different kinds of optimization problems in power systems field. To address this issue, this paper provides a comparative analysis of several evolutionary algorithms based on parametric and non-parametric statistical tests. Numerical examples are based on hydrothermal system operation and transmission pricing optimization problems.
\end{abstract}

Keywords- Evolutionary algorithm, hydrothermal system, transmission pricing.

\section{I.INTRODUCTION}

Due to their inherent complexity (e.g. non-linear, nonconvex, and multimodal search landscape), power system optimization problems do not always lend themselves to solution by using classical optimization algorithms. In view of this, there is an increased interest, especially in recent years, to develop solution approaches based on heuristic optimization algorithms [1]. Remarkably, most heuristic optimization algorithms offer conceptual simplicity, easy adaptability for application on different types of power system problems, and are open to further extensions, including the possibility of hybridization with classical optimization algorithms and other heuristic strategies (e.g. applied for local search).

Evolutionary optimization algorithms, and, particularly, genetic algorithm (GA) [2], particle swarm optimization (PSO) [3], and differential evolution (DE) [4], constitute the family of heuristic optimization algorithms which has received the greatest attention from researchers in power system field. These pioneer algorithms have been progressively extended in recent years to include especial strategies for adaptive parameter change, local search, re-initialization, adaptive population sizing, and population information exchange with the aim of enhancing the global search capability.

Despite these breakthroughs, the stochastic aspects (e.g. random initialization, random numbers used in the evolutionary operations) involved in the underlying evolutionary mechanism, and the computing effort (due to the fact that the evaluation of the fitness associated to each candidate solution might require computationally intensive computer simulations) motivate high concern on the effectiveness of these tools, especially when applied to solve high-dimensional optimization problems. Moreover, the development of optimization tools that perform successfully within a limited number of function evaluations is still an open and very challenging research issue.

Different groups of test functions have been proposed in existing literature, for instance, within the context of the IEEE-
CEC competitions [5]-[7], which are of great importance for evaluating and comparing the performance of modified or newly proposed algorithms. To date, the outcomes of several competitions highlight the potential of different emerging evolutionary algorithms to become generic optimization engines in the near future [8]. Nevertheless, whether these findings hold for power system optimization problems is still an open question, and constitutes the main motivation behind the work presented in this paper. It is worth pointing out that issues related to high-dimensionality, and performance within reduced computing effort, are out of the scope of this paper.

Based on hydrothermal system operation and transmission pricing optimization problems, this paper presents and discusses the outcomes of a comparative evaluation of the performance of seven recently developed evolutionary algorithms. The comparisons are based on statistical analysis (median, mean value, standard deviation) and non-parametric statistical tests (Wilcoxon, Friedman aligned rank test, and Quade test). The remainder of the paper is organized as follows: Section II overviews the definition of the studied power system optimization problems. In Section III, the main features of the compared evolutionary algorithms and the performed statistical analysis are presented. Section IV shows the experimental setup and provides a discussion on numerical results of the study. Concluding remarks and outline for further research are given in Section $\mathrm{V}$.

\section{POWER SYSTEM OPTIMIZATION PROBLEMS}

\section{A. Hydrothermal system operation}

The optimal hydrothermal system operation (OHSO) consists in determining the optimal combination of available generation sources to supply total system demand in economic way. Hence, it concerns minimization of the sum of production costs $t c_{\mathrm{i}}\left(g_{\mathrm{it}}\right)$ of $\mathrm{N}$ thermal units and non-supplied energy costs $c f_{\mathrm{k}}\left(g_{\mathrm{kt}}\right)$, for a given operational horizon comprising $\mathrm{N}_{\mathrm{s}}$ stages. The problem (ignoring losses) has the following format [9]: Minimize

$$
t c_{\text {total }}=\sum_{\mathrm{t}=1}^{\mathrm{Ns}}\left[\sum_{\mathrm{i}=1}^{\mathrm{N}} t c_{\mathrm{i}}\left(g_{\mathrm{it}}\right)+\sum_{\mathrm{k}=1}^{\mathrm{R}} c f_{\mathrm{k}}\left(g f_{\mathrm{kt}}\right)\right]
$$

subject to

- Energy balance Equation 


$$
\mathrm{d}_{\mathrm{t}}+\mathrm{p}_{\text {loss }}=\sum_{\mathrm{i}=1}^{\mathrm{N}} \mathrm{g}_{\mathrm{it}}+\sum_{\mathrm{j}=1}^{\mathrm{M}} \mathrm{ph}_{\mathrm{jt}+}+\sum_{\mathrm{k}=1}^{\mathrm{R}} \mathrm{gf}_{\mathrm{kt}}
$$

- Water Balance Equation

$x_{\mathrm{j}, \mathrm{t}+1}=x_{\mathrm{jt}}+y_{\mathrm{jt}}-q_{\mathrm{jt}}-s_{\mathrm{jt}}+\sum_{\mathrm{l}=1}^{\mathrm{L}}\left(q_{\mathrm{l},\left(\mathrm{t}-\tau_{\mathrm{d}}\right)}+s_{\mathrm{l},\left(\mathrm{t}-\tau_{\mathrm{d}}\right)}\right)$

- Physical limitations on reservoir storage volumes and discharge rates

$$
\begin{gathered}
\mathrm{x}_{\mathrm{j}, \text { min }}<x_{\mathrm{jt}}<\mathrm{x}_{\mathrm{j} \text {,max }} \\
\mathrm{q}_{\mathrm{j}, \text { min }}<q_{\mathrm{it}}<\mathrm{q}_{\mathrm{j}, \text { max }}
\end{gathered}
$$

- Loading limits

$$
\begin{gathered}
\mathrm{g}_{\mathrm{i}, \text { min }}<g_{\mathrm{jt}}<\mathrm{g}_{\mathrm{i}, \text { max }} \\
\mathrm{ph}_{\mathrm{j}, \text { min }}<p h_{\mathrm{jt}}<\mathrm{ph}_{\mathrm{j}, \text { max }}
\end{gathered}
$$

- Hydro Power Generation Equation

$$
\begin{gathered}
p h_{\mathrm{jt}}=\eta_{\mathrm{j}} h_{\mathrm{jt}} q_{\mathrm{jt}} \\
h_{\mathrm{j}, \mathrm{t}}=\phi\left(x_{\mathrm{jt}}\right)-\theta\left(u_{\mathrm{jt}}\right)-p c_{\mathrm{jt}}
\end{gathered}
$$

where:

$d_{\mathrm{t}} \quad$ : load demand in MW during stage $\mathrm{t}$

$p_{\text {loss }}$ : Losses in MW during stage $\mathrm{t}$

$g_{\text {it }}$ : Thermal generation in MW of unit i, during stage t.

$p h_{\mathrm{jt}}:$ hydro generation in MW of unit $\mathrm{j}$, during stage $\mathrm{t}$.

$g f_{\mathrm{kt}}$ : non-supplied generation in MW of node $\mathrm{k}$, during stage $\mathrm{t}$.

$y_{\mathrm{jt}} \quad$ : inflow rate for unit $\mathrm{j}$ in $\mathrm{hm}^{3} / \mathrm{s}$, during stage $\mathrm{t}$

$x_{\mathrm{jt}} \quad$ : storage in the reservoir $\mathrm{j} \mathrm{in} \mathrm{hm}^{3}$, during stage t.

$q_{\mathrm{jt}} \quad$ : turbine flow by unit $\mathrm{j}$ in $\mathrm{hm}^{3} / \mathrm{s}$, during stage $\mathrm{t}$.

$s_{\mathrm{jt}} \quad$ : spillage of unit $\mathrm{j}$ in $\mathrm{hm}^{3} / \mathrm{s}$, during stage $\mathrm{t}$.

$h_{\mathrm{jt}} \quad:$ average water head for unit $\mathrm{j}$ during stage $\mathrm{t}$.

$p c_{\mathrm{jt}}$ : hydraulic loss during stage $\mathrm{t}$.

$\eta_{j} \quad$ : productivity of plant $\mathrm{j}$, in $\frac{\mathrm{MW}}{\left(\mathrm{hm}^{3} / \mathrm{s}\right) * \mathrm{~m}}$

$\phi\left(x_{\mathrm{jt}}\right)$ : polynomial function of forebay volume-water head, in meters

$\theta\left(u_{\mathrm{jt}}\right):$ polynomial function of afterbay discharge-head, in meters

L : number of upstream units directly above $\mathrm{j}$ hydro plant.

M : number of hydro plants.

$\mathrm{N} \quad$ : number of thermal plants

$\tau_{\mathrm{d}} \quad$ : time delay to immediate downstream plant 1

$\mathrm{R}$ : node $\mathrm{k}$ with not supplied energy.

Ns : number of stages

In terms of an optimization problem, OHSO is described by:

- Inputs: load demand $d_{\mathrm{t}}$, water inflows $y_{\mathrm{jt}}$, limits $\mathrm{x}_{\mathrm{min}}$, $\mathrm{x}_{\max }, \mathrm{q}_{\min }, \mathrm{q}_{\max }, \mathrm{g}_{\min }, \mathrm{g}_{\max }, \mathrm{ph}_{\min }, \mathrm{ph}_{\max }$, productivity of plants $k_{\mathrm{jt}}$, and power generation functions $t c_{\mathrm{i}}\left(g_{\mathrm{it}}\right), c f_{\mathrm{k}}\left(g f_{\mathrm{kt}}\right), \phi\left(x_{\mathrm{jt}}\right), \theta\left(u_{\mathrm{jt}}\right)$.
- State variable: storage volumes $x_{\mathrm{jt}}$

- Control variables: thermal generation $g_{i t}$, non- supplied energy $g f_{\mathrm{kt}}$, spillage values $s_{\mathrm{jt}}$, and water discharges rates $q_{\mathrm{jt}}$

- Dependent variable: hydro generation $p h_{\mathrm{jt} .}$

\section{B. Pricing scheme}

The transmission pricing mechanism defined in [5] is adopted in this paper. Broadly speaking, it assigns some of the total charges due transmission system usage to bilateral customers, whereas the rest is distributed through pool customers. The first assignment is due to the fact that bilateral transactions are usually known a-priori, so the usage rate associated to them can be determined, for instance, by evaluating power transfer distribution factors (PTDs) [10]. By contrast, optimization is needed to properly decide equivalent bilateral exchanges such that the charges on pool customers are close to the bilateral transactions that have been absent.

It is assumed that an input file, containing bus and line specifications, fixed cost to be recovered and bilateral transactions data, is previously defined. Then, the principle of Equivalent Bilateral Exchange (EBE) is applied on the given information and the transmission charges are evaluated at each bus, such that the transmission usage rates for a generation at bus $i\left(T U_{g}^{i}\right)$ and a demand at bus $j\left(T U_{d}^{j}\right)$ are obtained. Next, equivalent bilateral transactions between pool generations and pool demands are obtained by minimizing usage rate deviations due to bilateral transactions. Mathematically, this optimization problem can be formulated as:

Minimize

$$
O F=\sum_{i}\left(\frac{F\left(G D_{i j}\right)}{P_{g i}-P_{g i}^{*}}-T U_{g}^{i}\right)^{2}+\sum_{j}\left(\frac{F\left(G D_{i j}\right)}{P_{d j}-P_{d j}^{*}}-T U_{d}^{j}\right)^{2}
$$

considering that

$$
F\left(G D_{i j}\right)=\frac{\mathrm{FC}^{k}}{\sum_{i} \sum_{j}\left|G D_{i j} \cdot \gamma_{i j}^{k}\right|+\sum_{i} \sum_{j}\left|\mathrm{BT}_{i j} \cdot \gamma_{i j}^{k}\right|} \sum_{j} G D_{i j} \sum_{k} \gamma_{i j}^{k}
$$

and subject to

$$
\begin{aligned}
& \sum_{i} G D_{i j}=P_{g i}-P_{g i}^{*} \quad \forall i \in \mathrm{N}_{\mathrm{g}} \\
& \sum_{j} G D_{i j}=P_{d j}-P_{d j}^{*} \quad \forall j \in \mathrm{N}_{\mathrm{d}}
\end{aligned}
$$

where $\mathrm{N}_{\mathrm{g}}$ and $\mathrm{N}_{\mathrm{b}}$ are the number of generator and load buses, respectively. $\mathrm{FC}^{k}$ stands for fixed cost of a line $k$ that needs to be recovered, $\mathrm{BT}_{i j}$ denotes bilateral transaction between generator at bus $i$ and demand at bus $j, P_{g i}$ is total generation at bus $i, P_{g i}^{*}$ is the sum of generations due to all bilateral transactions at bus $i, P_{d j}$ is the total demand at bus $j, P_{d j}^{*}$ is the sum of demands due to all bilateral transactions at bus $j$, and $G D_{i j}$ is the equivalent bilateral transaction that needs to be evaluated. $\gamma_{i j}^{k}$ denotes the sensitivity of a line $k$ connecting buses $m$ and $n$ for a transaction between buses $i$ and $j$, and is given by (5). 


$$
\gamma_{i j}^{k}=\frac{\mathrm{X}_{m i}-\mathrm{X}_{n i}-\mathrm{X}_{m j}-\mathrm{X}_{n j}}{\mathrm{X}_{m n}}
$$

where $\mathrm{X}_{m n}$ is the reactance of line connecting buses $m$ and $n$, whereas $\mathrm{X}_{m i}, \mathrm{X}_{n i}, \mathrm{X}_{m j}$, and $\mathrm{X}_{n j}$ are entries into the reactance matrix $\mathbf{X}$.

The set of optimization variables is defined by a vector containing all $G D_{i j}$. Each GD accounts for an exchange between a generator and a load in the system, so the size of the vector is $\mathrm{N}_{\mathrm{g}} \cdot \mathrm{N}_{\mathrm{b}}$. Therefore, the scale of the optimization problem increases with system size. Besides, the problem possesses a multimodal search space, so a powerful optimization solver is needed to find the most feasible solution. The lower bound for each $G D_{i j}$ is set to zero, whereas the upper bound is determined by using (16).

$$
\max \left\{G D_{i j}\right\}=\min \left\{P_{g i}-\mathrm{BT}_{i j}, P_{d j}-\mathrm{BT}_{i j}\right\}
$$

Once the best values of GDs are found, the usage rates for pool generations and pool demands can be easily calculated by using simple expressions, which are functions of GDs and can be found in [5]. They are not reproduced here due to space constraints.

\section{COMPARISON CONSIDERATIONS}

Among the selected algorithms for the comparative study are the hybrid variant of the mean-variance mapping optimization (MVMO-SH) [11], the genetic algorithm with multi-parent crossover (GA-MPC) [2], the comprehensive learning particle swarm optimization (CLPSO) [12], the covariance matrix adaptation evolution strategy (CMA-ES) [13], the adaptive differential evolution algorithm with variable population size (JADE-vPS) [14], the self-adaptive differential evolution algorithm (SADE) [15], and the linearized biogeography-based optimization (LBBO) [16]. These tools are recent additions to metaheuristic optimization field, and they have proven an excellent performance when applied to different benchmark optimization problems.

Due to space constraint, thorough description of each algorithm is not presented in this paper. Committed readers can find details in the above-mentioned references. Fig. 1 illustrates the general procedure followed by any of these algorithms. Broadly speaking, the performance of each algorithm is affected by randomness in the initialization stage and some factors involved in the evolutionary operators (e.g. parent selection and mutation), which entails that different results are obtained each time the algorithm runs. Besides, the main difference between the algorithms resides in the way the evolution operations are performed.

Due to their stochastic nature, it is crucial to run multiple repetitions (e.g. Monte Carlo simulation) of the optimization when comparing evolutionary algorithms. The collected outcomes from each repetition allow preliminary comparisons, for instance, in terms of the average performance, the variance/standard deviation, best-case performance, worst-case performance, of the best achieved fitness value. Note however, that these statistical metrics depend on the number of repetitions performed and the degree of randomness. Thus, it might happen that, on average, the algorithm A1 performs better than the algorithm A, but the opposite could be happen if the experiment is performed with the same or a different number of repetitions [17].

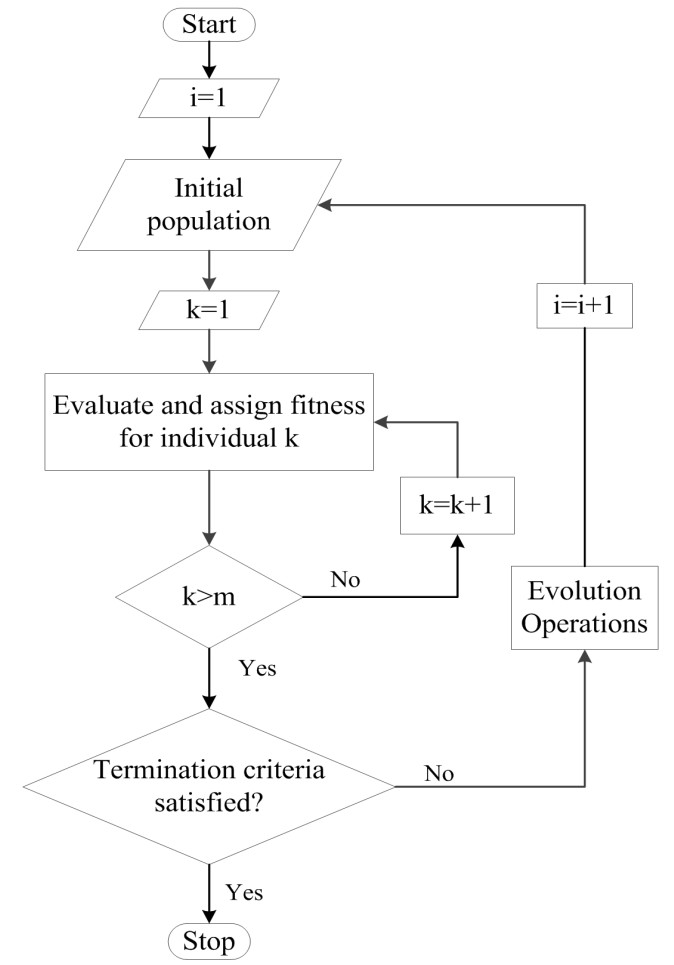

Fig. 1. Simplified diagram of the hydro-thermal test system

Different types of statistical tests have been proposed in existing literature to improve the evaluation process in a broader context. These procedures are categorized into parametric and non-parametric test [17]. Parametric tests (e.g. ttest) are based on certain assumptions, such as independence, normality, and homoscedasticity, which are frequently violated. By contrast, non-parametric tests are suitable when the aforesaid assumptions cannot be satisfied, especially in multiproblem analysis. Thus, based on the guidelines provided in [18], the following non-parametric statistical tests are considered in this paper, aiming at detecting significant behavior differences between the compared algorithms: Wilcoxon rank sum tests, Friedman aligned rank test, and Quade test.

\section{IV.RESULTS}

A small-size system with four hydro power plants and one thermal power plant, whose details are given in [19], was used for the comparisons concerning the OHSO problem. The IEEE 30 bus benchmark system was used for the transmission pricing optimization problem. Details of system data as well as lower and upper limits for optimization variables can be found in [5].

Numerical experiments were performed on a Dell personal computer equipped with Intel ${ }^{\circledR}$ Core $^{\top \mathrm{TM}}$ i7 $4600 \mathrm{U}$ CPU, 2.70 GHz and 8 GB RAM, under Windows 7 enterprise, 64 bit OS. The implementation of the optimization task and the 
evolutionary algorithms was done in Matlab ${ }^{\circledR}$ Version R2014b. For each algorithm, the fitness $f^{*}$ is calculated as follows:

$$
\begin{gathered}
f^{*}=f+\mathrm{K}+p_{1}+p_{2} \\
p_{1}=\sum_{i=1}^{N_{\text {icon }}} \rho_{i}^{\dagger} \cdot \max \left[0, g_{i}(\mathbf{x})\right] \\
p_{2}=\sum_{j=1}^{N_{\text {econ }}} \rho_{j}^{\dagger \dagger} \cdot \max \left[0,\left|h_{j}(\mathbf{x})\right|-\varepsilon\right]
\end{gathered}
$$

where $f$ is the objective function value, $\mathbf{x}$ is the vector of optimization variables (candidate solution or particle), $\mathrm{N}_{\text {icon }}$ is the number of inequality constraints, $\mathrm{N}_{\text {econ }}$ is the number of equality constraints. Furthermore, $g_{i}$ denotes the i-th inequality constraint, and $h_{\mathrm{j}}$ the j-th equality constraint. $\rho_{i}^{\dagger}$ and $\rho_{j}^{\dagger \dagger}$ stand for penalty coefficient (factor) for each constraint, which were set to the values provided in [5]. For equality constraints, the tolerance parameter is set to $\varepsilon=0.0001$. The constant $\mathrm{K}$ is automatically set either to zero (if there is no constraint violation) or to the highest fitness value among all particles in the first evaluation round (step). The aim of this approach is to give always preference to feasible solutions, which is crucial in the initial stage of the search process, where there is a high possibility of having unfeasible solutions. A thorough assessment considering more sophisticated constraint handling techniques is beyond the scope of this paper. The purpose is rather to ascertain the effectiveness of each algorithm when used closer to its pure form.

Each algorithm was tuned by accounting the typical values and ranges provided in the references mentioned above and by performing sensitivity analysis of the achieved fitness value under a single parameter change within 10 independent optimization runs in all problems. The parameter settings used for each algorithm are listed below:

- MVMO-SH: $\mathrm{N}_{\mathrm{p}}=150$, archive $\operatorname{size}=5, \quad \gamma_{\mathrm{LS}}=0$, $g_{\mathrm{p}_{-} \text {ini }}^{*}=0.7, \quad g_{\mathrm{p}_{-} \text {final }}^{*}=0.2, \quad \mathrm{~m}_{\text {ini }}=\mathrm{D} / 4, \quad \mathrm{~m}_{\text {final }}=1$, $\Delta \mathrm{d}_{0}=0.2, f_{\mathrm{s} \_ \text {ini }}^{*}=1$, and $f_{\mathrm{s} \_ \text {final }}^{*}=10$.

- GA-MPC: $\mathrm{Np}=100 \quad$ (population size); $\mathrm{CR}=1$ (crossover rate), $\beta=\mathrm{N}(0.7,0.1)$, archive pool size $=$ $0.5^{*} \mathrm{~N}_{\mathrm{p}}$, and $\mathrm{p}=0.1$ (diversity operator).

- CLPSO: $\mathrm{N}_{\mathrm{p}}=40 ; \mathrm{c}=1.50$ (acceleration constant), and $\mathrm{w}=0.9$ (inertia weight). The learning probability for each particle $i$ was assigned by using

$$
P c_{i}=0.05+0.45\left(\frac{\exp \left(\frac{10(i-1)}{\mathrm{N}_{\mathrm{p}}}\right)-1}{\exp \left(\mathrm{N}_{\mathrm{p}}\right)-1}\right)
$$

- CMA-ES: $\mathrm{N}_{\mathrm{p}}=100$. Only this parameter was tuned, whereas the remaining parameters were initialized by following the procedure explained in [13].
- JADE-VPS: $\mathrm{N}_{\mathrm{p}}=40, \mathrm{~F}=0.8$ (mutation factor), $\mathrm{CR}=0.5$.

- SADE: $\mathrm{N}_{\mathrm{p}}=50, \mathrm{~F}=\mathrm{N}(0.5,0.3), \mathrm{CR}_{\mathrm{m}}=0.5$ (median of $\mathrm{CR})$, learning period $=70$.

- LBBO: $\mathrm{N}_{\mathrm{p}}=40, \mathrm{p}_{\mathrm{m}}=0.01$ (mutation probability), $\mathrm{k}_{\mathrm{p}}=2$ (elitism parameter), $\mathrm{N}_{\text {grad }}=2$ (best individuals to use local search); $\mathrm{g}_{\mathrm{dimp}}=0.1$ (improvement threshold for gradient descent), $\mathrm{R}_{\text {ini }}=1000$ (re-initialization rate), $\mathrm{LH}_{\mathrm{p}}=1000$ (points in Latin hypercube for local search).

Statistical tests on convergence performance and quality of final solution provided by each algorithm were performed by executing 30 independent optimization runs. Uniform random initialization within the search space is adopted and the random seed is based on time. Each optimization run is terminated upon completion of 150,000 function evaluations.

A comparative summary concerning the obtained best (minimum), worst (maximum), median, mean, and standard deviation of fitness in the last iteration, is presented in Table 1, including the outcomes of Wilcoxon rank sum test, which were performed by taking MVMO-SH as reference for pairwise comparison with other algorithms. The results of the test are represented by $h$ values in the Table, where $h=1$ indicates that the algorithms are statistically different with 95\% certainty. Friedman aligned rank test and Quade test were also carried out in order to further evaluate and compare the performance of MVMO-SH and the other selected algorithms. The outcomes of these tests are summarized in Table 2. The following remarks are summarized from Tables 1 and 2:

- The best results for both optimization problems were achieved by using MVMO-SH, closely followed by CMA-ES and JADE-VPS. The statistics achieved by using these algorithms for solving this problem are quite similar, whereas there are significant differences with the statistics achieved by using the remaining algorithms.

- The performance of all algorithms was statistically different in both optimization problems. MVMO-SH achieves the best rank (i.e. best performing algorithm) in the Friedman aligned and Quade tests. The p-values computed through statistics of each of the tests suggests the existence of significant differences among the compared algorithms.

\section{CONCLUSIONS}

This paper provides a comparative statistical analysis between seven recently developed evolutionary (metaheuristic) algorithms. Despite of differences in their evolutionary mechanisms, all methods were initialized with a random population, and then performed iteratively to create new candidate solutions, a procedure that also entails random factors in the evolutionary operations. The algorithms were empirically tuned based on algorithm's sensitivity to a single parameter change. Numerical results showed that MVMO-SH, CMA-ES and JADE-VPS achieved the best performance among all compared algorithms when tackling hydrothermal system operation and transmission pricing optimization problems. Further research will consider a more systematic tuning of the 
algorithms as well as higher dimensional problems in order to ascertain if the conclusions drawn in this paper hold for large scale variants of the studied problems.

Table 1. RANKS ACHIEVED BY FRIEDMAN ALIGNED AND QUADE TESTS

\begin{tabular}{|c|c|c|c|c|c|c|c|c|}
\hline Problem & Parameter & GA-MPC & CLPSO & CMA-ES & JADE-VPS & SADE & LBBO & MVMO-SH \\
\hline \multirow{5}{*}{ OHSO } & Best & $9.5267 \mathrm{e}+05$ & $9.4274 \mathrm{e}+05$ & $9.3992 \mathrm{e}+05$ & $9.3710 \mathrm{e}+05$ & $9.3716 \mathrm{e}+05$ & $9.3361 \mathrm{e}+05$ & $9.3363 \mathrm{e}+05$ \\
\cline { 2 - 9 } & Worst & $1.5407 \mathrm{e}+06$ & $1.8651 \mathrm{e}+06$ & $9.5360 \mathrm{e}+05$ & $9.4658 \mathrm{e}+05$ & $1.1684 \mathrm{e}+06$ & $2.5621 \mathrm{e}+06$ & $9.4474 \mathrm{e}+05$ \\
\cline { 2 - 9 } & Median & $9.7249 \mathrm{e}+05$ & $9.4752 \mathrm{e}+05$ & $9.4708 \mathrm{e}+05$ & $9.4255 \mathrm{e}+05$ & $9.4351 \mathrm{e}+05$ & $9.5018 \mathrm{e}+05$ & $9.4085 \mathrm{e}+05$ \\
\cline { 2 - 9 } & Mean & $1.0073 \mathrm{e}+06$ & $1.0375 \mathrm{e}+06$ & $9.4669 \mathrm{e}+05$ & $9.4263 \mathrm{e}+05$ & $9.6357 \mathrm{e}+05$ & $1.0703 \mathrm{e}+06$ & $9.4052 \mathrm{e}+05$ \\
\cline { 2 - 9 } & Std. & $1.2404 \mathrm{e}+05$ & $2.2018 \mathrm{e}+05$ & $3.4853 \mathrm{e}+03$ & $2.0679 \mathrm{e}+03$ & $5.8404 \mathrm{e}+04$ & $3.3180 \mathrm{e}+05$ & $2.3414 \mathrm{e}+03$ \\
\cline { 2 - 9 } & h & 1 & 1 & 1 & 1 & 1 & 1 & --- \\
\hline \multirow{5}{*}{ Pricing } & Best & $8.9583 \mathrm{e}+00$ & $1.0150 \mathrm{e}+01$ & $6.7641 \mathrm{e}+00$ & $6.7730 \mathrm{e}+00$ & $1.1735 \mathrm{e}+01$ & $1.2941 \mathrm{e}+01$ & $6.7641 \mathrm{e}+00$ \\
\cline { 2 - 9 } & Worst & $8.7808 \mathrm{e}+01$ & $6.8141 \mathrm{e}+01$ & $6.7682 \mathrm{e}+00$ & $7.8468 \mathrm{e}+00$ & $2.7511 \mathrm{e}+01$ & $3.8086 \mathrm{e}+02$ & $6.7644 \mathrm{e}+00$ \\
\cline { 2 - 9 } & Median & $1.8818 \mathrm{e}+01$ & $1.7237 \mathrm{e}+01$ & $6.7641 \mathrm{e}+00$ & $6.9340 \mathrm{e}+00$ & $1.6678 \mathrm{e}+01$ & $1.0317 \mathrm{e}+02$ & $6.7640 \mathrm{e}+00$ \\
\cline { 2 - 8 } & Mean & $2.8111 \mathrm{e}+01$ & $1.8705 \mathrm{e}+01$ & $6.7644 \mathrm{e}+00$ & $7.0240 \mathrm{e}+00$ & $1.7047 \mathrm{e}+01$ & $1.2295 \mathrm{e}+02$ & $6.7641 \mathrm{e}+00$ \\
\cline { 2 - 8 } & Std. & $2.1859 \mathrm{e}+01$ & $1.0031 \mathrm{e}+01$ & $8.5401 \mathrm{e}-04$ & $2.8282 \mathrm{e}-01$ & $3.1732 \mathrm{e}+00$ & $8.3827 \mathrm{e}+01$ & $7.5154 \mathrm{e}-05$ \\
\cline { 2 - 8 } & h & 1 & 1 & 1 & 1 & 1 & -- \\
\hline
\end{tabular}

Table 2. RANKS ACHIEVED BY FRIEDMAN ALIGNED AND QUADE TESTS

\begin{tabular}{|c|c|c|}
\hline Algorithm/test & Friedman aligned & Quade \\
\hline MVMO-SH & 107.4 & 2.62 \\
\hline GA-MPC & 141.25 & 2.79 \\
\hline LBBO & 188.31 & 3.97 \\
\hline CMA-ES & 206.27 & 4.13 \\
\hline SADE & 224.23 & 4.64 \\
\hline CLPSO & 226.44 & 5.39 \\
\hline JADE-vPS & 232.79 & 5.32 \\
\hline Statistic & 41.190 & 22.30 \\
\hline p-value & $7.84 \mathrm{e}-04$ & $6.54 \mathrm{e}-09$ \\
\hline
\end{tabular}

\section{VI.REFERENCES}

[1] J.L. Rueda, and I. Erlich, "Mean-Variance Mapping Optimization for the Solution of Power System Problems," in Proc. 17th International Conference on Intelligent System Applications to Power Systems, Tokyo, Japan, July 2013.

[2] S.M.Elsayed, R.A.Sarker, and D.L. Essam, "A new genetic algorithm for solving optimization problems," Engineering Applications of Artificial Intelligence, vol. 27, pp. 57-69, Jan. 2014.

[3] V.S. Pappala, "Application of PSO for Optimization of Power Systems under Uncertainty," $\mathrm{PhD}$ dissertation, University Duisburg-Essen, Duisburg, Germany, 2009.

[4] S. Das, and P.N. Suganthan,"Differential Evolution: A Survey of the State-of-the-Art," IEEE Transactions on Evolutionary Computation, vol. 15, no. 1, pp. 4-31, Feb. 2011.

[5] S. Das, and P. N. Suganthan. (2010, Dec.). Problem Definitions and Evaluation Criteria for CEC 2011 Competition on Testing Evolutionary Algorithms on Real World Optimization Problems. [Online]. Available: http://www.ntu.edu.sg/home/epnsugan/.

[6] B. Liu, Q. Chen, Q. Zhang, J.J. Liang, P. N. Suganthan, and B.Y. Qu, "Problem Definitions and Evaluation Criteria for Computational Expensive Optimization,” Technical Report, Dec. 2013. [Online]. Available at: http://www.ntu.edu.sg/home/epnsugan/

[7] J.J. Liang1, B.Y. Qu, and P.N. Suganthan, "Problem Definitions and Evaluation Criteria for the CEC 2015 Competition on Learning-based Real-Parameter Single Objective Optimization," Technical Report, Nanyang Technological University (Singapore) and Zhengzhou
University (China), Nov. 2013. [Online]. Available at: http://www.ntu.edu.sg/home/epnsugan/

[8] P.N. Suganthan, "Testing Evolutionary Algorithms on Real-World Numerical Optimization Problems". [Online]. Available at: http://www.ntu.edu.sg/home/epnsugan/

[9] A.A.F.M. Carnero. "Contrubição ao Planejamento da Operação Energética de Sistemas Hidrotérmicos de Potencia”. Tese de Doutorado, Unicamp, Capinas, 1991.

[10] R.D. Christie, B.F. Wollenberg, and I. Wangensteen, "Transmission management in the deregulated environment," Proceedings of the IEEE, vol.88, no.2, pp.170-195, Feb. 2000

[11] I. Erlich, J.L. Rueda, and S. Wildenhues, "Evaluating the Mean-Variance Mapping Optimization on the IEEE-CEC 2014 Test Suite", in Proc. 2014 IEEE World Congress on Computational Intelligence, July 2014.

[12] J.J. Liang, A.K. Qin, P.N. Sugantha, S. Baskar, "Comprehensive learning particle swarm optimizer for global optimization of multimodal functions," IEEE Transactions on Evolutionary Computation, vol. 10, no. 3, pp. 281- 295, June 2006.

[13] N. Hansen. (2011, June). The CMA Evolution Strategy: A Tutorial. [Online]. Available: https://www.lri.fr/ $\sim$ hansen/cmatutorial1 10628.pdf

[14] W. Nakawiro, and I. Erlich, "A new adaptive differential evolution algorithm for voltage stability constrained optimal power flow," in Proc. 17 th Power Systems Computation Conference, Stockholm, Sweden, Aug. 2011.

[15] A.K. Qin, V.L. Huang, P.N. Suganthan, "Differential Evolution Algorithm With Strategy Adaptation for Global Numerical Optimization," IEEE Transactions on Evolutionary Computation, vol. 13, no. 2, pp. 398 - 417, April 2009.

[16] D. Simon, M.G.H. Omran, M. Clerc, "Linearized Biogeography-Based Optimization with Re-initialization and Local Search," Information Sciences, vol. 267, pp. 140-157, May 2014.

[17] D. Simon, Evolutionary Optimization Algorithms: Biologically Inspired and Population-Based Approaches to Computer Intelligence. Hoboken: John Wiley \& Sons, 2013.

[18] Derrac J, García S, Molina D, Herrera F. A practical tutorial on the use of nonparametric statistical tests as a methodology for comparing evolutionary and swarm intelligence algorithms. Swarm and Evolutionary Computation. March 2011; 1(1): 3-18.

[19] J. Labaide. "Optimal Operation of Multireservoir Systems: State-of-theArt Review". Journal of Water Resources Planning and Management ASCE, March-April 2004, pp 93-111. 